\title{
COLLISIONS BETWEEN HIGH LATITUDE CLOUDS: THEORY MEETS OBSERVATIONS
}

\author{
J.C. Lattanzio ${ }^{1}$ and E.R. Keto ${ }^{2}$ \\ ${ }^{1}$ Institute of Geophysics and Planetary Physics \\ ${ }^{2}$ Lawrence Livermore National Laboratory
}

We report on fully three-dimensional hydrodynamic and radiative transfer simulations of collisions between high latitude clouds. Our model uses the smoothed particle hydrodynamics code described by Lattanzio and Henriksen (1988) to compute the velocity, temperature, and density fields in the impacted clouds and a recently developed radiative transfer code to compute ${ }^{13} \mathrm{CO}$ line radiation from the simulated source. By including the instrumental effects involved in a particular observations we can make detailed comparisons with the observations. The model shows that: 1) The previously unexplained energy source for the broad $C O$ line wings reported by Blitz, Magnani, and Wandel (1988) derives from the collisions. 2) Collisions can induce rapid gravitational collapse and star formation in these clouds which are otherwise supported against gravitational contraction via their internal energy content. 3) The external pressure due to intercloud HI, firts proposed for these objects by Keto and Myers (1986), plays a significant role in the stability and evolution of the high latitude clouds. 